\title{
Cutting Down the Energy Consumed by Domestic Robots: Insights from Robotic Vacuum Cleaners
}

\author{
Florian Vaussard, Philippe Rétornaz, David Hamel, and Francesco Mondada \\ EPFL - STI - LSRO \\ Station 9, 1015 Lausanne, Switzerland \\ firstname. lastname@epfl.ch
}

\begin{abstract}
The market of domestic service robots, and especially vacuum cleaners, has kept growing during the past decade. According to the International Federation of Robotics, more than one million units were sold worldwide in 2010. Currently, there is no in-depth analysis of the energetic impact of the introduction of this technology on the mass market. This topic is of prime importance in our energy-dependant society. This study aims at identifying key technologies leading to the reduction of the energy consumption of a domestic mobile robot, by exploring the design space using technologies issued from the robotic research field, such as the various localization and navigation strategies. This approach is validated through an in-depth analysis of seven vacuum-cleaning robots. These results are used to build a global assessment of the influential parameters. The major outcome is the assessment of the positive impact of both the ceiling-based visual localization and the laser-based localization approaches.
\end{abstract}

\section{Motivation}

This study aims at analysing the impact of some research results on the energy consumption of mobile domestic robots, with a focus on localization and navigation. Such robots, and especially vacuum-cleaning robots, have become widespread. IRobot, one of the top companies on this market, claims to have sold six million units of its "Roomba" robot since the first release in 2002 [1]. In the present study, we analysed several domestic robots. They have been selected to represent the various technologies used today in robotics, and some of them include new features on the mass market, such as visual localization.

The minimization of the energy consumption for such devices is an important and broad topic to be considered, especially for such a growing mass-market and within our energy-dependant society. A domestic robot should ideally be able to operate autonomously indoors, without having to connect to the power grid. This implies to embed the energy harvesters into the mobile robot or on a charging station, providing it with energy extracted directly from the surrounding environment, as previously discussed in [12]. The primary source of energy can be for example light, heat, or mechanical work produced by humans, or any combination of them. In any case, the available energy level is low and highly fluctuating, driving the need to spare energy at the level of the complete system.

Therefore this paper presents an analysis of the performances of several existing products, assessing the impact of the embedded technologies on energy consumption. 


\subsection{Working Hypotheses}

The total energy $\mathrm{E}_{\text {total }}$ consumed by the system, expressed as a function of a set of generalized design parameters $\vec{\alpha}$, can be written as in Eq. 1 , where $\eta_{\text {charger }}$ is the efficiency of the charging electronics, $T_{\text {task }}$ is the time needed to complete the task, and $p_{\text {robot }}(t)$ is the instantaneous power of the robot.

$$
E_{\text {total }}(\vec{\alpha})=\frac{1}{\eta_{\text {charger }}} \int_{0}^{T_{\text {task }}(\vec{\alpha})} p_{\text {robot }}(t, \vec{\alpha}) d t
$$

In this study, we want to explore the design space $\vec{\alpha}$ in order to minimize $\mathrm{E}_{\text {total }}(\vec{\alpha})$. This can be achieved by using two strategies: either by reducing $p_{\text {robot }}(t)$ over time, or by reducing $T_{\text {task }}$.

The instantaneous power $p_{\text {robot }}(t)$ comes from the "useful" power on one side, and from the losses on the other side. The required power is minimized by removing useless functions or fusing together several functions, leading for example to a decrease in the number of motors used. Losses are minimized by increasing the robot's efficiency, for example by reducing the numerous electrical and magnetic losses inside the motors, as well as by reducing the Joule losses inside the electronics. For a mobile robot, the energy lost when braking also accounts for a part of the total losses, and it can be partially recovered by the addition of appropriate electronics. The overall control, such as obstacle avoidance, is equally important, in order to follow a smooth trajectory and avoid unnecessary brakings.

Another strategy is the minimization of the completion time $T_{\text {task }}$. In this case, it is often useless to increase the robot's speed, because it will increase the instantaneous power accordingly. Better planning and navigation are the key for this strategy to succeed. When complete coverage is required, as in cleaning, patrolling, or lawn-mowing tasks, a path planning coupled with a localization strategy will cut down the coverage time, compared to a random walk approach. Recent developments in the semiconductor industry for mobile applications, coupled with algorithmic and mechatronic advances such as the laser scanner of [8], have made the simultaneous localization and mapping (SLAM) affordable for the mass market.

Reducing $T_{\text {task }}$ often comes at the price of extra sensors and computational power, needed to achieve an efficient localization, which conflicts with the reduction of the instantaneous power of the first strategy. In this article, we will mainly assess the effect of the navigation strategy on the total energy, based on measures performed with real mobile domestic robots.

\subsection{Mobile Robots for Domestic Environments}

Currently in the domestic environment, only a few mobile robots have been mass-produced. The first successful product, and now the most widespread, is the vacuum cleaner, with first researches dating back to the 1980s [13], while the first prototype for domestic use can be dated back to 1991 [11]. Up to now, studies have compared mobile domestic robots only from an historical or purely technical point of view $[6,10]$. 
Other commercial applications include lawn-mowing, telepresence, pool, or gutter cleaning [2]. In the literature, other examples like assistive [3] or rehabilitation robotics [4] can also be found.

Most of the researche has focused on aspects like the navigation in dynamic environments [15] or the social acceptance [14]. The question of the energy-efficiency was only considered lately, mainly pointing out the lack of regulations and standards, compared to other home appliances [5]. Other researchers studied performance metrics, such as the coverage of several domestic mobile robots performing a random walk [9]. The present study can be seen as an extension to robots performing more advanced navigation strategies, and with the energy as the final consideration.

From the literature, it can be seen that the impact of some new technologies on energy consumption remains yet unexplored. This study proposes to fill in the gap.

\section{Experimental Analysis}

The analysis was done using several robotic vacuum cleaners. After briefly explaining our methodology in Sec. 2.1, following sections will detail the results.

\subsection{Methodology}

This study is based on a sample of seven robots, ranging from the low-cost derivatives of the "Roomba" robot, to recent products embedding more complex sensors and algorithms and costing up to $600 \$$. Robots 1 to 3 follow a random walk, using some predefined behaviours (wall following, spirals, obstacle avoidance...). Robots 4 to 6 perform Ceiling Visual SLAM (CV-SLAM), implementing an algorithm similar to the one described by [7]. The last one is fitted with a low-cost laser range scanner, performing 2D Laser SLAM [8]. A detailed description is available in Table 1, at the end of this article.

We explored several performance metrics, in relation with the energy. Both the global and instantaneous powers were measured during realistic scenarios. The evolution of the coverage as a function of the time was also measured using an overhead camera.

The experimental setup is depicted in Fig. 1. It recreates a two-room flat with a total surface of $15.5 \mathrm{~m}^{2}$. A precision power analysis bench was used to measure the overall power drawn by the charging station, whereas we used a wireless datalogger to measure the robot's in situ power $p_{\text {robot }}(t)$.

\subsection{Power Analysis}

An in situ analysis of the consumed power was first performed, by placing an embedded datalogger module between the battery and the robot itself. This module records precisely the power consumed by the robot, during each step of the process. Statistical results can be found in Table 2, at the end of this article. Informative plots are drawn in Fig. 2. 


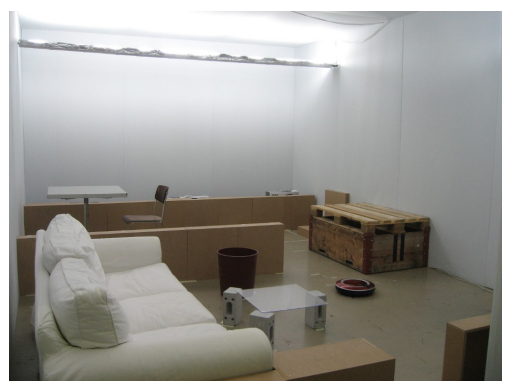

(a) Side view.

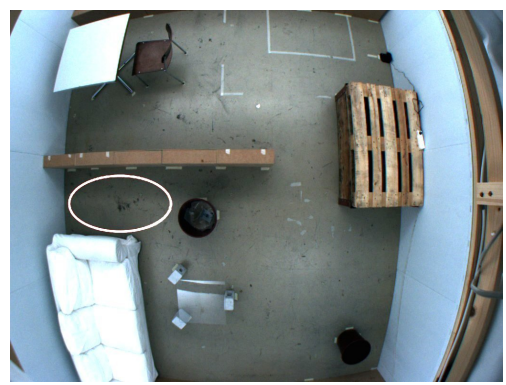

(b) View from the overhead camera. The white ellipse marks an area of difficult access.

Fig. 1: The experimental setup.

The robots performing CV-SLAM all use a dual-layer architecture, as revealed by a detailed analysis of the electronics; a low-power controller is in charge of the sensors, the motors, and other low-level functions, while an embedded processor performs the image analysis and the subsequent pose estimation.

On Fig. 2a, the startup sequence of the cleaning process for Robot 7 (laser SLAM) is clearly visible. Starting from the idle state, the following phases can be identified: 1) the laser's spinning motor starts and stabilises; 2) the powerful suction fan starts; 3 ) the main brush starts to rotate; 4) finally the robot starts the driving motors and begins to clean. It can be deduced that the laser SLAM itself consumes about $1.9 \mathrm{~W}(6.3 \%$ of the total cleaning power), compared to the cleaning subsystem, which takes $23.8 \mathrm{~W}$ $(78.8 \%)$. The mobility account only for $2.5 \mathrm{~W}(8.3 \%)$. For this specific robot, the power used for the navigation functions is marginal compared to the cleaning aperture.

Such a clear breakdown is however not always visible, for example in the case of Robot 5 (Fig. 2b). The first small increase of power (black circle) is devised to be due to the visual SLAM subsystem (camera and algorithm). It takes about $1.1 \mathrm{~W}$ of extra power, $8.4 \%$ of the total power. All the motors start together.

Fig. 3 plots the distribution of the power consumption of each robot for several cases. Let us first consider the idle case, when the robot is turned on, but not moving. The three robots performing CV-SLAM are, not surprisingly, among the top consumers, as the embedded processor will need between 0.5 to $1 \mathrm{~W}$ of extra power, even when not processing any images.

When considering the cleaning cases, things are completely different. The previous increase, due to the extra processing power, is largely overwhelmed by the difference due to the driving and cleaning motors. Thus, the addition of the SLAM represents only a small part of the total consumption, when compared to the energy required for moving and cleaning. As we will see in Sec. 2.3, SLAM-enabled robots benefit from the speed-up of coverage, saving energy on the overall process.

A power analysis was also done directly at the plug of the recharge station. Complete results are in Table 3, at the end of this article. A first discovery is the high idle 


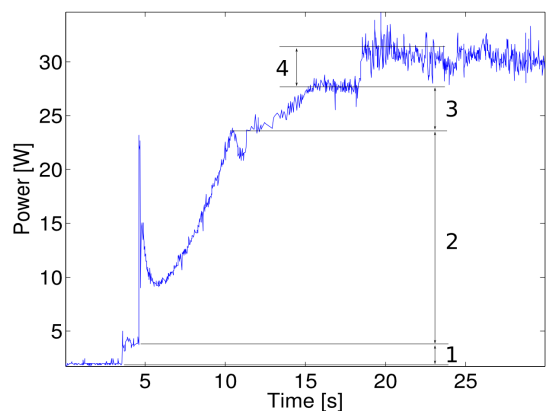

(a) Robot 7: Carpet cleaning startup processes. 1: laser 2: suction 3: brush 4: driving motors.

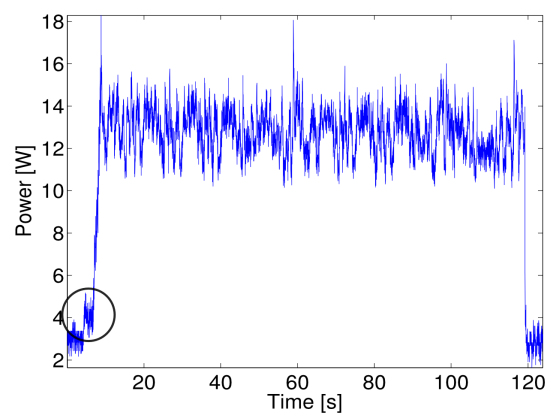

(b) Robot 5: Cleaning on concrete. The black circle pinpoints the startup of the CVSLAM process, just before the robot starts moving.

Fig. 2: Plots of the in situ power measures.

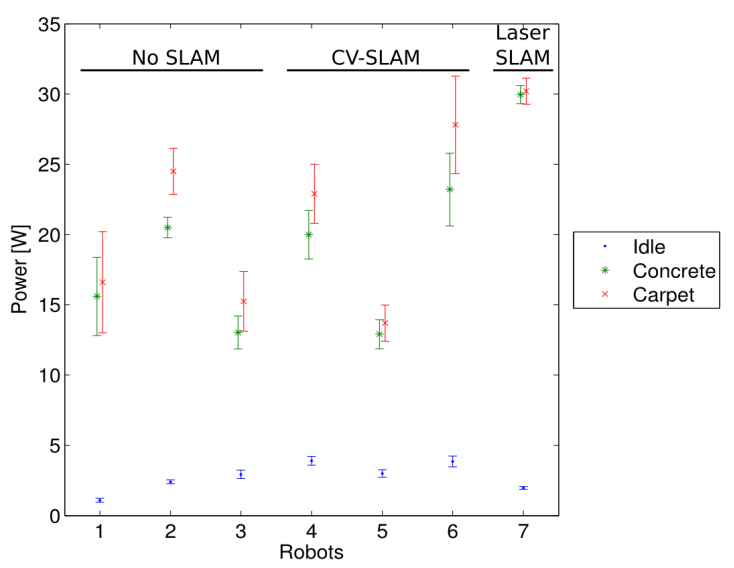

Fig. 3: Task-related in situ power measured for each robot. More than 1000 samples have been used for each data.

power of the base station, up to $3.5 \mathrm{~W}$ for the worst result (Robot 2). This is even worse when the robot is connected to the base station. Even fully charged, the power consumption is between 3.2 and $8.1 \mathrm{~W}$. Unfortunately, this kind of appliance is not bound by the European regulation $1275 / 2008$, currently limiting the standby mode to $2 \mathrm{~W}$. This represents a serious concern for such mass-produced electrical appliances.

The efficiency of the recharge station $\eta_{\text {charger }}$ was computed as

$$
\eta_{\text {charger }}=\frac{1}{E_{\text {total }}} \int_{0}^{T_{\text {task }}} p_{\text {robot }}(t) d t
$$


where $E_{\text {total }}$ is the energy consumed at the plug to recharge the robot, and $p_{\text {robot }}(t)$ is the in situ power measured on the robot during the whole process.

This figure of efficiency includes the intrinsic quality of the charger, as well as the efficiency of the battery subsystem, and vary between 0.33 and 0.84 in our study. The top two robots are not surprisingly the ones using Li-ion batteries. Others use the $\mathrm{Ni}-\mathrm{MH}$ technology. In the case of two Ni-MH powered robots, more than $50 \%$ of the recharge power is lost.

\subsection{Coverage Analysis}

A cleaning robot, like some other domestic robots, should not only be low-power and energy-efficient, but should also effectively cover the area to clean. Using a video tracking system, we have recorded the trajectories when cleaning the flat of Fig. 1a. A sample of each trajectory is shown in Fig. 4. It is very informative about the strategies taken by each robot.

The evolution of the average coverage, as a function of the time, is plotted in Fig. 5. The SLAM-enabled robots are much faster than the others, which is confirmed by the completion time shown in Fig. 6. Robot 6, which is the slowest among the robots performing SLAM, is still three times faster than the random-walk fellows.

Regarding the total coverage, the SLAM-enabled Robot 4, and in a less measure Robot 5, underperform compared to the others. Looking back at the image analysis, it appears that some places are harder to reach. One of these places is between the sofa, the intermediate wall, and the bin (white ellipse in Fig. 1b). In about $50 \%$ of the runs, robots 4 and 5 were unable to reach this place, losing accordingly part of the coverage. On the contrary, Robot 6 was $100 \%$ successful on a total of 11 runs, as its path planning uses thinner bands, as one can see in Fig. 4f, compared to the coarser displacements of the two others. While some time is lost by this strategy, it gains greatly in robustness.

\subsection{Final Comparison}

The central question of this study is the influence of design parameters on the energy consumption, and especially the navigation strategy. To answer this question, we now proceed to a comparison of the coverage strategy, with respect to the energy. For this, we define the specific energy, which is the energy needed to cover $1 \mathrm{~m}^{2}$ of floor. It equals

$$
E_{\text {specific }}=\frac{1}{A_{\text {effective }}} \int_{0}^{T_{\text {task }}} p_{\text {robot }}(t) d t
$$

where $A_{\text {effective }}$ is the surface effectively covered, as deduced from the previous coverage analysis. Fig. 7 clearly shows the effectiveness of the SLAM-enabled robots over random walk methods, counterbalancing the increase of power by a reduced $T_{\text {task }}$. However, no clear conclusion can be drawn between CV-SLAM and Laser SLAM robots. 


\section{Random navigation}

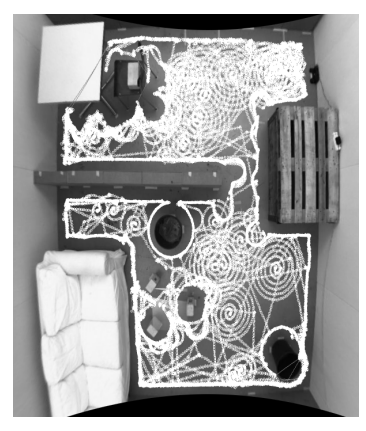

(a) Robot 1

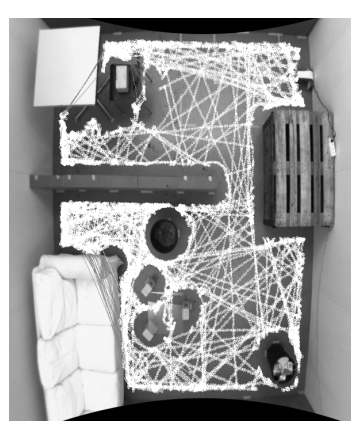

(b) Robot 2

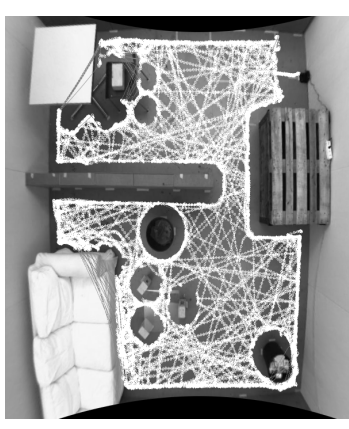

(c) Robot 3

\section{Ceiling Visual SLAM}

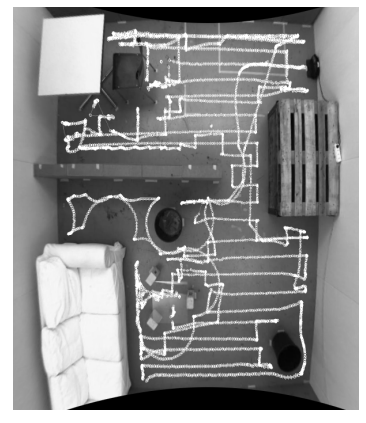

(d) Robot 4

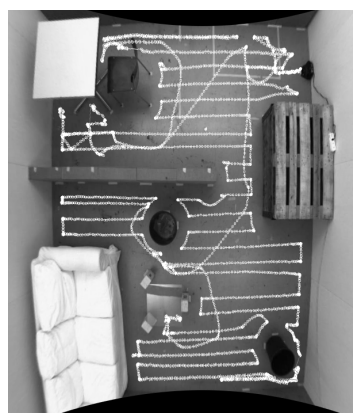

(e) Robot 5

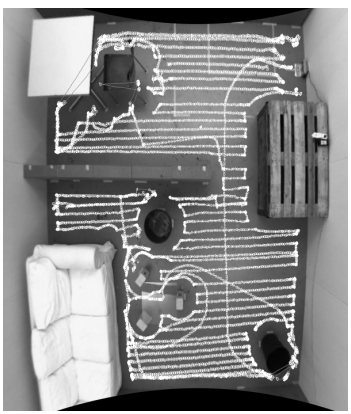

(f) Robot 6

\section{Laser SLAM}

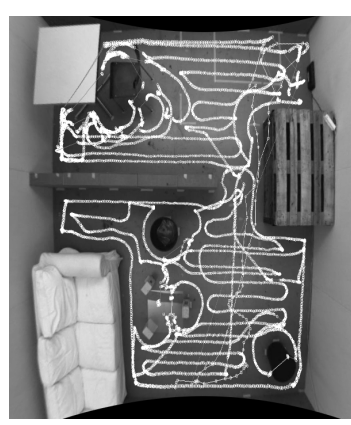

(g) Robot 7

Fig. 4: Sample of the trajectories. 


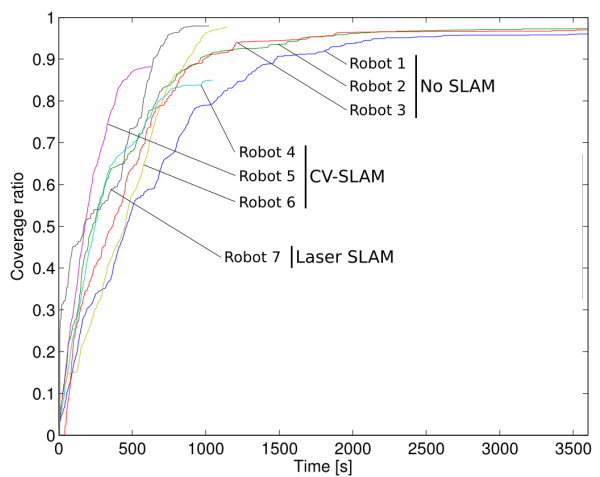

Fig. 5: Evolution of the average coverage over one hour.

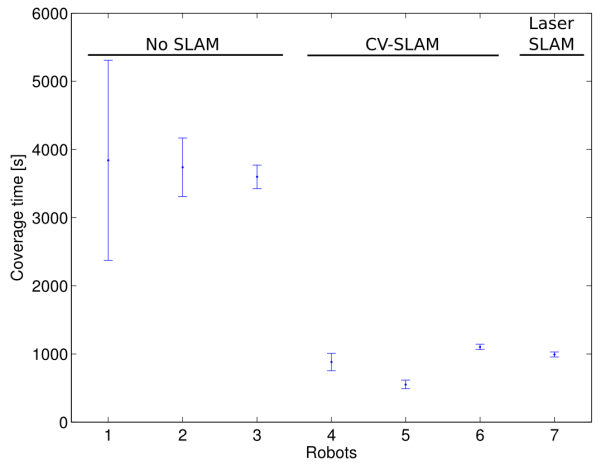

Fig. 6: Cleaning time for each robot.

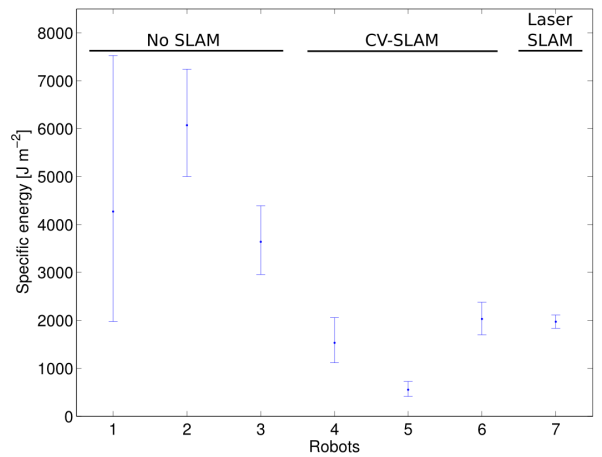

Fig. 7: The specific energy, in $\mathrm{J} \mathrm{m}^{-2}$, for each robot. 


\section{Conclusion}

This study has presented a methodological evaluation of the energy-efficiency of representative vacuum-cleaning robots, with the aim of designing energy-efficient domestic mobile robots in the future.

If we take a systemic approach, the whole energy chain has to be considered, starting from the charging up to its final usage. As noticed in this study, the efficiency of commercial charging stations is rather low, with important losses in the electronics. Liion batteries, when properly used, also benefit from the efficiency of the energy storage. But as they are more expensive compared to Ni-MH technology, and need dedicated recharge electronics, their adoption is for now limited in such devices.

Based on in situ and global power measurements of existing products, the influence of some technologies could be determined. From this analysis, it is determined that robots required to fully cover an area have a clear advantage, from the energy point of view, if they can rely on an embedded SLAM system. Ceiling Visual SLAM and Laser SLAM are clearly advantageous for energy consumption: they reduce the coverage time, and they have a relatively small impact on the instantaneous power. However, no conclusion could be drawn between the two SLAM systems.

Regarding the total coverage, some of the SLAM-enabled robots underperformed compared to the others. This results from the path planning strategy, as some locations can be missed if only a coarse displacement is performed. It is believed that such problems are a matter of fine tuning the system. To add a visual SLAM-based navigation into a product, the price is mainly driven by the cost of the processor and camera boards. As the smartphone market expands, the cost of such components is rapidly decreasing. Old processors, like the iMX.31 used by Robot 5, can now be negotiated for less than $10 \$$.

The energy taken by the displacement and the cleaning aperture are much higher than the localization subsystem. The reduction of the energy consumption will come also through an optimization of the mechatronics. For example, Robot 5 uses only one motor to drive the three brushes, compared to the three motors needed by robots 4 and 6. Moreover, the idle consumption is clearly not optimised, leading to huge losses if the device remains connected all day long. The effect of a braking energy recovery device should also be studied, as the kinetic energy is for now definitively lost when breaking.

Acknowledgments. This research was supported by the Swiss National Science Foundation through the National Centre of Competence in Research Robotics. Most of the robots have been provided by the Swiss National Television (TSR). We also thank Daniel Burnier for the datalogger used during the in situ analysis.

\section{References}

1. iRobot CEO Discusses Q4 2010 Results http://seekingalpha.com/article/ 252090-irobot-ceo-discusses-q4-2010-results-earnings-calltranscript

2. Alonso, I.: Service robotics. Service Robotics within the Digital Home pp. 89-114 (2011) 
3. Breuer, T., Macedo, G.R.G., Hartanto, R., Hochgeschwender, N., Holz, D., Hegger, F., Jin, Z., Müller, C., Paulus, J., Reckhaus, M., Ruiz, J.A.Á., Plöger, P.G., Kraetzschmar, G.K.: Johnny: An Autonomous Service Robot for Domestic Environments. Journal of Intelligent and Robotic Systems 66(1-2), 245-272 (2012)

4. Carrera, I., Moreno, H.A., Saltarén, R.J., Pérez, C., Puglisi, L., Cena, C.E.G.: ROAD: domestic assistant and rehabilitation robot. Med. Biol. Engineering and Computing 49(10), 1201-1211 (2011)

5. De Almeida, A., Fong, J.: Domestic Service Robots [TC Spotlight]. Robotics \& Automation Magazine, IEEE 18(3), 18-20 (2011)

6. Fiorini, P., Prassler, E.: Cleaning and Household Robots: A Technology Survey. Auton. Robots 9(3), 227-235 (2000)

7. Jeong, W., Lee, K.: CV-SLAM: A new ceiling vision-based SLAM technique. In: Intelligent Robots and Systems, 2005.(IROS 2005). 2005 IEEE/RSJ International Conference on. pp. 3195-3200. IEEE (2005)

8. Konolige, K., Augenbraun, J., Donaldson, N., Fiebig, C., Shah, P.: A low-cost laser distance sensor. In: ICRA. pp. 3002-3008. IEEE (2008)

9. Palleja, T., Tresanchez, M., Teixido, M., Palacín, J.: Modeling floor-cleaning coverage performances of some domestic mobile robots in a reduced scenario. Robotics and Autonomous Systems 58(1), 37-45 (2010)

10. Prassler, E., Kosuge, K.: Domestic Robotics. In: Siciliano, B., Khatib, O. (eds.) Springer Handbook of Robotics, pp. 1253-1281. Springer (2008)

11. Prassler, E., Ritter, A., Schaeffer, C., Fiorini, P.: A Short History of Cleaning Robots. Auton. Robots 9(3), 211-226 (2000)

12. Vaussard, F., Bonani, M., Rétornaz, P., Martinoli, A., Mondada, F.: Towards autonomous energy-wise RObjects. Towards Autonomous Robotic Systems pp. 311-322 (2011)

13. Yasutomi, F., Yamada, M., Tsukamoto, K.: Cleaning robot control. In: Robotics and Automation, 1988. Proceedings., 1988 IEEE International Conference on. pp. 1839-1841. IEEE (1988)

14. Young, J.E., Hawkins, R., Sharlin, E., Igarashi, T.: Toward Acceptable Domestic Robots: Applying Insights from Social Psychology. I. J. Social Robotics 1(1), 95-108 (2009)

15. Yuan, F., Twardon, L., Hanheide, M.: Dynamic path planning adopting human navigation strategies for a domestic mobile robot. In: IROS. pp. 3275-3281. IEEE (2010) 

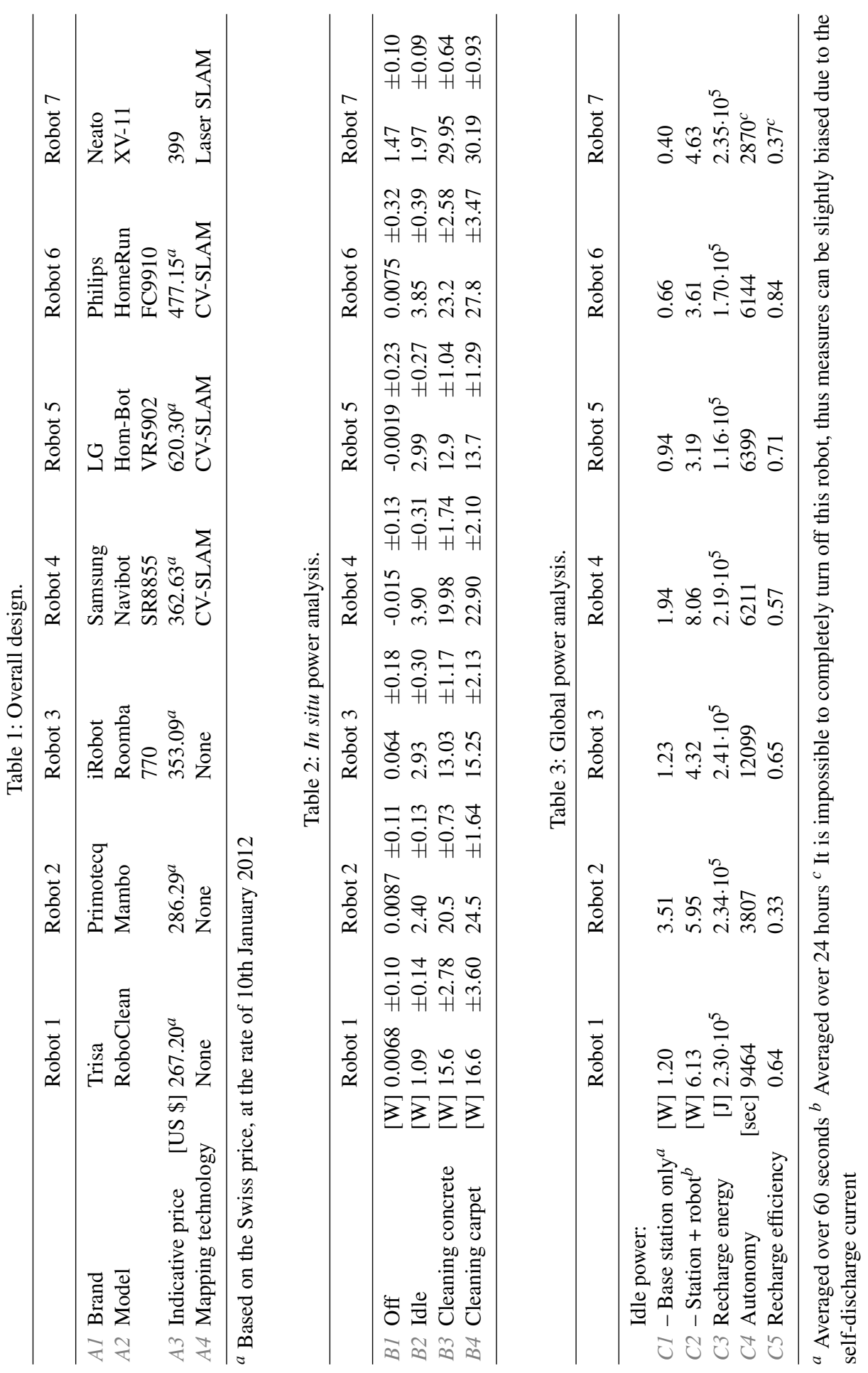


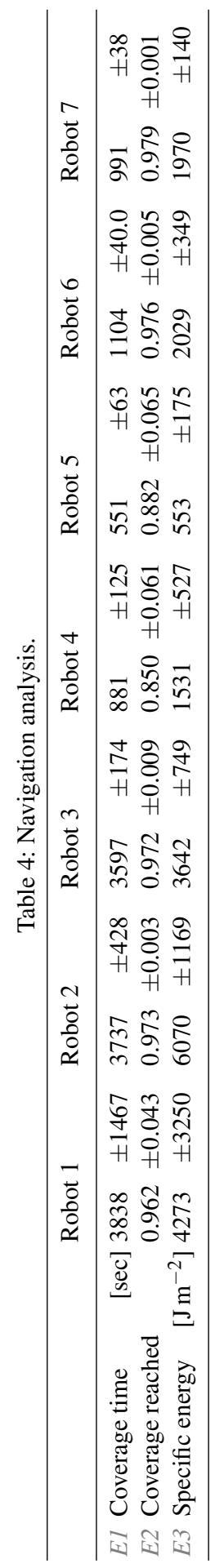

